PROCEEDINGS OF THE

AMERICAN MATHEMATICAL SOCIETY

Volume 139, Number 12, December 2011, Pages 4225-4234

S 0002-9939(2011)10900-6

Article electronically published on April 28, 2011

\title{
DERIVED EQUIVALENCE INDUCED BY INFINITELY GENERATED $n$-TILTING MODULES
}

\author{
SILVANA BAZZONI, FRANCESCA MANTESE, AND ALBERTO TONOLO
}

(Communicated by Harm Derksen)

\begin{abstract}
Let $T_{R}$ be a right $n$-tilting module over an arbitrary associative ring $R$. In this paper we prove that there exists an $n$-tilting module $T_{R}^{\prime}$ equivalent to $T_{R}$ which induces a derived equivalence between the unbounded derived category $\mathcal{D}(R)$ and a triangulated subcategory $\mathcal{E}_{\perp}$ of $\mathcal{D}\left(\operatorname{End}\left(T^{\prime}\right)\right)$ equivalent to the quotient category of $\mathcal{D}\left(\operatorname{End}\left(T^{\prime}\right)\right)$ modulo the kernel of the total left derived functor $-\otimes_{S^{\prime}}^{\mathbb{L}} T^{\prime}$. If $T_{R}$ is a classical $n$-tilting module, we have that $T=T^{\prime}$ and the subcategory $\mathcal{E}_{\perp}$ coincides with $\mathcal{D}$ (End $\mid(T)$ ), recovering the classical case.
\end{abstract}

\section{INTRODUCTION}

Tilting theory generalizes the classical Morita theory of equivalences between module categories. Originated in the works of Gel'fand and Ponomarev, Brenner and Butler, Happel and Ringel [6, 9, 19, it has been generalized in various directions. In the recent literature, given an associative ring $R$ with $0 \neq 1$, a right $R$-module $T_{R}$ (possibly infinitely generated) is said to be $n$-tilting if the following conditions are satisfied:

(T1) there exists a projective resolution of right $R$-modules

$$
0 \rightarrow P_{n} \rightarrow \ldots \rightarrow P_{1} \rightarrow P_{0} \rightarrow T \rightarrow 0
$$

(T2) $\operatorname{Ext}_{R}^{i}\left(T, T^{(\alpha)}\right)=0$ for each $i>0$ and each cardinal $\alpha$;

(T3) there exists a coresolution of right $R$-modules

$$
0 \rightarrow R \rightarrow T_{0} \rightarrow T_{1} \rightarrow \ldots \rightarrow T_{m} \rightarrow 0
$$

where the $T_{i}$ 's are direct summands of arbitrary direct sums of copies of $T$.

If the projectives $P_{i}$ 's in (T1) can be assumed finitely generated, then the $n$-tilting module $T_{R}$ is said to be classical n-tilting.

Infinitely generated tilting modules arise naturally; they are objects of interest in themselves and also in the context of representation theory of Artin algebras. Relevant examples are studied in [28, 24, 2]. Moreover, they play an important role in connection with homological conjectures. In [3] it is proved that the little finitistic dimension of a Noetherian ring is finite if and only if there is an $n$-tilting

Received by the editors February 18, 2010 and, in revised form, September 9, 2010 and October 21,2010 .

2010 Mathematics Subject Classification. Primary 16D90, 18E30, 18 E35.

This research was supported by grant CPDA071244/07 of Padova University and MIUR PRIN 2007.

(C)2011 American Mathematical Society

Reverts to public domain 28 years from publication 
module representing in a canonical way the category of finitely presented modules of finite projective dimension. Even in the case of finite dimensional algebras it could be possible that this tilting module is necessarily infinitely generated.

Let us denote by $S=\operatorname{End}\left(T_{R}\right)$ the endomorphism ring of $T$ and by $K E_{i}(T)$ and $K T_{i}(T), 0 \leq i \leq n$, the following classes:

$$
\begin{gathered}
K E_{i}(T)=\left\{M \in \operatorname{Mod}-R: \operatorname{Ext}_{R}^{j}(T, M)=0 \text { for each } 0 \leq j \neq i\right\}, \\
K T_{i}(T)=\left\{N \in \operatorname{Mod}-S: \operatorname{Tor}_{j}^{S}(N, T)=0 \text { for each } 0 \leq j \neq i\right\} .
\end{gathered}
$$

In 1986 Miyashita 27] proved that if $T_{R}$ is a classical $n$-tilting, then the functors $\operatorname{Ext}_{R}^{i}(T,-)$ and $\operatorname{Tor}_{i}^{S}(-, T)$ induce equivalences between the classes $K E_{i}(T)$ and $K T_{i}(T)$.

In the same year, works of several authors showed that the natural context for studying equivalences induced by classical tilting modules is that of derived categories. In particular Cline, Parshall and Scott [10, generalizing a result of Happel [18, proved that a classical $n$-tilting module $T_{R}$ provides a derived equivalence between the bounded derived categories $\mathcal{D}^{b}(R)$ and $\mathcal{D}^{b}(S)$ of bounded cochain complexes of right $R$ - and $S$-modules.

In the context of infinite dimensional tilting theory, Facchini 12, 13, in 1988 proved that, over a commutative domain, the divisible module $\partial$ introduced by Fuchs 14 is an infinitely generated 1-tilting module and it provides a pair of equivalences

$$
K E_{0}(\partial) \underset{-\otimes \partial}{\stackrel{\operatorname{Hom}(\partial,-)}{\rightleftarrows}} K T_{0}(\partial) \cap I-C o t, \quad K E_{1}(\partial) \underset{\operatorname{Tor}_{1}(-, \partial)}{\stackrel{\operatorname{Ext}^{1}(\partial,-)}{\rightleftarrows}} K T_{1}(\partial) \cap I-C o t
$$

between the category $K E_{0}(\partial)$ of all divisible modules and the category $K T_{0}(\partial) \cap$ $I$-Cot of all $I$-reduced $I$-cotorsion modules, and the category $K E_{1}(\partial)$ of all reduced modules and the category $K T_{1}(\partial) \cap I$-Cot of all I-divisible $I$-cotorsion modules, respectively. This equivalence generalizes both the Harrison and Matlis equivalences 20, 26. In 1995 Colpi and Trlifaj [11 started the study in general of 1-tilting modules. They realized that it can be useful to "change slightly" the tilting module to realize a good equivalence theory. They proved that if $T_{R}$ is a 1-tilting module, there exists another 1-tilting module $T_{R}^{\prime}$ equivalent to $T_{R}$ (i.e. $K E_{0}(T)=K E_{0}\left(T^{\prime}\right)$ ), with endomorphism ring $S^{\prime}=\operatorname{End}\left(T^{\prime}\right)$, such that the functors $\operatorname{Hom}_{R}\left(T^{\prime},-\right)$ and $-\otimes_{S^{\prime}} T^{\prime}$ induce an equivalence between $K E_{0}(T)=K E_{0}\left(T^{\prime}\right)$ and its image class in Mod- $S^{\prime}$. Moreover $T^{\prime}$ results in a finitely presented $S^{\prime}$-module. In 2001 Gregorio and Tonolo extended this result proving the existence of a pair of equivalences

$$
K E_{i}\left(T^{\prime}\right) \underset{\operatorname{Tor}_{i}^{S^{\prime}}\left(-, T^{\prime}\right)}{\stackrel{\operatorname{Ext}_{R}^{i}\left(T^{\prime},-\right)}{\rightleftarrows}} K T_{i}\left(T^{\prime}\right) \cap \operatorname{Cost}\left(T^{\prime}\right), \quad i=0,1
$$

where $\operatorname{Cost}\left(T^{\prime}\right)$ is the class of costatic right $S^{\prime}$-modules (see [17]).

In 2009 Bazzoni [5] gives a better understanding of the whole situation in the setting of derived categories proving that for a 1-tilting module $T_{R}$ it is possible to find an equivalent 1-tilting module $T^{\prime}$ which induces a derived equivalence between the unbounded derived category $\mathcal{D}(R)$ and the quotient category of $\mathcal{D}\left(S^{\prime}\right)$ modulo the full triangulated subcategory $\operatorname{Ker}\left(-\otimes_{S^{\prime}}^{\mathbb{L}} T^{\prime}\right)$, namely the kernel of the total left derived functor of the functor $-\otimes_{S^{\prime}} T^{\prime}$. 
In this paper we generalize Bazzoni's result to a general $n$-tilting module $T_{R}$. We prove the existence of a good $n$-tilting module $T_{R}^{\prime}$ equivalent to $T_{R}$ (see Definition 1.1], which, also in such a case, provides a derived equivalence between the unbounded derived category $\mathcal{D}(R)$ and a triangulated subcategory $\mathcal{E}_{\perp}$ of $\mathcal{D}\left(\operatorname{End}\left(T^{\prime}\right)\right)$. The category $\mathcal{E}_{\perp}$ turns out to be equivalent to the quotient category of $\mathcal{D}\left(\operatorname{End}\left(T^{\prime}\right)\right)$ modulo the kernel of the total left derived functor $-\otimes_{S^{\prime}}^{\mathbb{L}} T^{\prime}$. Moreover, as done in 25 in the contravariant case, we interpret the derived equivalence at the level of stalk complexes obtaining on the underlying module categories a generalization of the Miyashita equivalences.

\section{1. n-TILTING CLASSES}

In 2004 Bazzoni (see [4) proved that $T_{R}$ is an $n$-tilting module if and only if the classes

$$
T^{\perp \infty}:=\left\{M_{R}: \operatorname{Ext}_{R}^{i}(T, M)=0 \text { for each } i>0\right\}
$$

and

$$
\operatorname{Gen}_{n}(T):=\left\{M_{R}: \exists T^{\left(\alpha_{n}\right)} \rightarrow \ldots \rightarrow T^{\left(\alpha_{1}\right)} \rightarrow M \rightarrow 0, \text { for some cardinals } \alpha_{i}\right\}
$$

coincide.

Definition 1.1. Two $n$-tilting right $R$-modules $T_{R}$ and $T_{R}^{\prime}$ are said to be equivalent if $\operatorname{Gen}_{n}\left(T_{R}\right)=\operatorname{Gen}_{n}\left(T_{R}^{\prime}\right)$.

An arbitrary direct sum of copies of an $n$-tilting module is an $n$-tilting module equivalent to the original one. Therefore equivalent tilting modules can have completely different endomorphism rings.

Definition 1.2. We say that $T_{R}$ is a $\operatorname{good} n$-tilting module if it is $n$-tilting and it satisfies the condition

$\left(\mathrm{T} 3^{\prime}\right)$ there is an exact sequence

$$
0 \rightarrow R \rightarrow T_{0} \rightarrow T_{1} \rightarrow \ldots \rightarrow T_{n} \rightarrow 0
$$

where the $T_{i}$ 's are direct summands of finite direct sums of copies of $T$.

It is easy to verify that a classical $n$-tilting module is good (see e.g. [16, p. 189]).

Proposition 1.3. For any $n$-tilting module $T_{R}$ there exists an equivalent good $n$ tilting module $T_{R}^{\prime}$ such that

$$
K E_{i}(T)=K E_{i}\left(T^{\prime}\right) \text { for each } i \geq 0 .
$$

Proof. Let $T_{R}$ be an $n$-tilting module. If it is classical, then $T$ already satisfies (T3'). Otherwise, from condition (T3) let us consider $T^{\prime}=T_{0} \oplus \ldots \oplus T_{n}$. Since $T^{\prime}$ is a direct summand of a direct sum of copies of $T$, we have

$$
\operatorname{Gen}_{n}\left(T^{\prime}\right) \subseteq \operatorname{Gen}_{n}(T)=T^{\perp_{\infty}} \subseteq T^{\perp_{\infty}},
$$

and $T^{\prime}$ satisfies properties (T1) and (T2) of tilting modules. Since by construction it satisfies also property $\left(\mathrm{T} 3^{\prime}\right)$, we have $\operatorname{Gen}_{n}\left(T^{\prime}\right)=T^{\perp_{\infty}}$ and $T^{\prime}$ is the wanted good $n$-tilting module equivalent to $T$.

Finally, since $\operatorname{Ker}_{\operatorname{Ext}^{j}}(T,-)=\operatorname{Ker}_{\operatorname{Ext}^{j}}\left(T_{0} \oplus \ldots \oplus T_{n},-\right)=\operatorname{Ker}_{\operatorname{Ext}^{j}}\left(T^{\prime},-\right)$, we conclude that $K E_{i}(T)=K E_{i}\left(T^{\prime}\right)$ for each $i \geq 0$. 
A good $n$-tilting module has an endomorphism ring $S$ sufficiently large to permit building a good equivalence theory between the unbounded derived categories $\mathcal{D}(R)$ and $\mathcal{D}(S)$. In the sequel we will work directly with good $n$-tilting modules.

Proposition 1.4. Let $T_{R}$ be a good n-tilting module and $S=\operatorname{End}\left(T_{R}\right)$. Then ${ }_{S} T$ has a projective resolution

$$
0 \rightarrow Q_{n} \rightarrow \ldots \rightarrow Q_{0} \rightarrow{ }_{S} T \rightarrow 0,
$$

where the $Q_{i}$ 's are direct summands of a finite direct sum of copies of $S, \operatorname{Ext}_{S}^{i}(T, T)$ $=0$ for each $i \geq 0$, and $R \cong \operatorname{End}\left({ }_{S} T\right)$.

Proof. By Definition 1.2 there is an exact sequence

$$
0 \rightarrow R \rightarrow T_{0} \rightarrow T_{1} \rightarrow \ldots \rightarrow T_{n} \rightarrow 0
$$

with the $T_{i}$ 's direct summands of $T^{m}$ for a suitable $m \in \mathbb{N}$. Denote by $K_{i}$ the kernel of the map $T_{i} \rightarrow T_{i+1}, 1 \leq i \leq n-1$. Applying the contravariant functor $\operatorname{Hom}_{R}(-, T)$ we get easily by dimension shifting that

$$
0=\operatorname{Ext}_{R}^{i}\left(K_{j}, T\right) \text { for each } 1 \leq j \leq n-1 \text {, and } i \geq 1 .
$$

Therefore we have the exact sequence

$$
\begin{aligned}
0 \rightarrow \operatorname{Hom}_{R}\left(T_{n}, T\right) \rightarrow \operatorname{Hom}_{R}\left(T_{n-1}, T\right) \rightarrow \ldots \rightarrow & \operatorname{Hom}_{R}\left(T_{1}, T\right) \\
& \rightarrow \operatorname{Hom}_{R}\left(T_{0}, T\right) \rightarrow{ }_{S} T \rightarrow 0,
\end{aligned}
$$

where each $\operatorname{Hom}_{R}\left(T_{i}, T\right)$ is a direct summand of $\operatorname{Hom}_{R}\left(T^{m}, T\right)=S^{m}$ and hence a finitely generated projective $S$-module. Given a right $R$-module $M$, let us denote for simplicity by $M^{*}$ the left $S$-module $\operatorname{Hom}_{R}(M, T)$, by $M^{* *}$ the right $R$-module $\operatorname{Hom}_{S}\left(M^{*}, T\right)$, and by $\delta_{M}$ the evaluation map $M \rightarrow M^{* *}$. The modules $K_{i}^{*}$ are the cokernels of the morphisms $\operatorname{Hom}_{R}\left(T_{i+1}, T\right) \rightarrow \operatorname{Hom}_{R}\left(T_{i}, T\right), 1 \leq i \leq n-$ 1. Applying to $(\dagger)$ the contravariant functor $\operatorname{Hom}_{S}(-, T)$ we get the following commutative diagrams with exact rows:

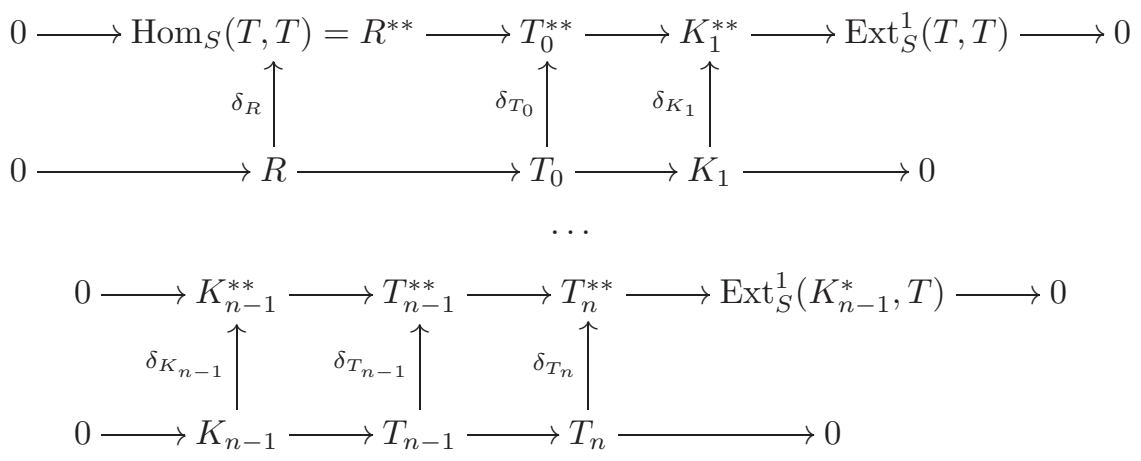

Since the $\delta_{T_{i}}$ 's are isomorphisms we get

$\operatorname{Ext}_{S}^{1}(T, T)=0$ and $0=\operatorname{Ext}_{S}^{1}\left(K_{i}^{*}, T\right) \cong \operatorname{Ext}_{S}^{i+1}(T, T)$ for each $1 \leq i \leq n-1$, and $R \cong \operatorname{Hom}_{S}(T, T)$.

Lemma 1.5 (Lemmas 1.8, 1.9 27]). Let $T_{R}$ be a good $n$-tilting and $S=\operatorname{End} T$. For any right $R$-module $M$ in $T^{\perp_{\infty}}$ and any right projective $S$-module $P_{S}$, we have

(1) $\operatorname{Tor}_{i}^{S}\left(\operatorname{Hom}_{R}(T, M), T\right)=0$ for each $i>0$;

(2) $\operatorname{Hom}_{R}(T, M) \otimes_{S} T \cong M, \quad f \otimes t \mapsto f(t)$; 
(3) $\operatorname{Ext}_{R}^{i}\left(T, P \otimes_{S} T\right)=0$ for each $i>0$.

If $T_{R}$ is a classical $n$-tilting module, then

(4) $P \cong \operatorname{Hom}_{R}\left(T, P \otimes_{S} T\right), \quad p \mapsto(f: t \mapsto p \otimes t)$.

Proof. Everything except condition (3) follows by the quoted lemmas in [27]. If $P \leq{ }^{\oplus} S^{(\alpha)}$ we have

$$
\operatorname{Ext}_{R}^{i}\left(T, P \otimes_{S} T\right) \leq{ }^{\oplus} \operatorname{Ext}_{R}^{i}\left(T, S^{(\alpha)} \otimes_{S} T\right)=\operatorname{Ext}_{R}^{i}\left(T, T^{(\alpha)}\right)=0 .
$$

\section{Tilting equivalences in DeRived CATegories}

In the sequel, for any ring $R$, we denote by $\mathcal{K}(R)$ the homotopy category of unbounded complexes of right $R$-modules and by $\mathcal{D}(R)$ the associated derived category. Given an object $M \in \operatorname{Mod}-R$, we continue to denote by $M$ also the stalk complex in $\mathcal{D}(R)$ associated to $M$, i.e. the complex with $M$ concentrated in degree zero. Any complex $C^{\bullet} \in \mathcal{D}(R)$ admits a $K$-injective resolution, i.e. a complex $\underline{\mathbf{i}} C^{\bullet}$ quasi-isomorphic to $C^{\bullet}$ whose terms are injective modules such that $\operatorname{Hom}_{\mathcal{K}(R)}\left(N^{\bullet}, \underline{\mathrm{i}} C^{\bullet}\right)=0$ for each exact complex $N^{\bullet}$. Similarly, any complex $C^{\bullet} \in \mathcal{D}(R)$ admits a $K$-projective resolution, i.e. a complex $\underline{\mathbf{p}} C^{\bullet}$ quasi-isomorphic to $C^{\bullet}$ whose terms are projective modules such that $\operatorname{Hom}_{\mathcal{K}(R)}\left(\underline{\mathbf{p}} C^{\bullet}, N^{\bullet}\right)=0$ for each exact complex $N^{\bullet}$ (see for instance [7, 22]). This result guarantees the existence of the total derived functor of any additive functor defined on module categories.

Given any covariant left exact functor $H: \operatorname{Mod}-R \rightarrow \operatorname{Mod}-S$, we denote by $\mathbb{R} H$ its total right derived functor defined on $\mathcal{D}(R)$. For any $C^{\bullet} \in \mathcal{D}(R), \mathbb{R} H\left(C^{\bullet}\right)$ coincides with the complex $H\left(\underline{\mathbf{i}} C^{\bullet}\right)$, where we still denote by $H$ its extension to $\mathcal{K}(R)$. Similarly, for any right exact covariant functor $G: \operatorname{Mod}-S \rightarrow \operatorname{Mod}-R$, we denote by $\mathbb{L} G$ its total left derived functor defined on $\mathcal{D}(S)$. For any $N^{\bullet} \in \mathcal{D}(S)$, $\mathbb{L} G\left(N^{\bullet}\right)$ coincides with the complex $G\left(\underline{\mathbf{p}} N^{\bullet}\right)$.

A module $M$ in Mod- $R$ is called $H$-acyclic if $R^{i} H M:=H^{i}(\mathbb{R} H M)=0$ for any $i \neq 0$. The abelian group $R^{i} H M$ coincides with the usual $i$-th derived functor $H^{(i)}(-)$ of $H$ evaluated in $M$. Analogously $G$-acyclic objects are defined and $L^{i} G(-):=H^{i}(\mathbb{L} G(-))=G^{(-i)}(-)$. Following the proof of [21, Corollary I.5.3. $\gamma$ ], in case the functor $H$ has finite homological dimension, the class $\mathfrak{J}$ of the complexes with $H$-acyclic components satisfies the conditions 1 and 2 of [21, Theorem I.5.1]; therefore for any complex $M^{\bullet}$ in $\mathcal{D}(R)$, we have

$$
\mathbb{R} H M^{\bullet}=H\left(J^{\bullet}\right),
$$

where $J^{\bullet}$ is a complex in $\mathfrak{J}$ quasi-isomorphic to $M^{\bullet}$. The analogous result holds for the left derived functor of $G$, in case $G$ has finite homological dimension.

In view of these considerations, by Lemma 1.5 we have the following result:

Corollary 2.1. Let $T_{R}$ be a good n-tilting module with endomorphism ring $S$. Then for each injective module $I_{R}$ and each projective module $P_{S}$ we have

(1) $\operatorname{Hom}_{R}(T, I)$ is $-\otimes_{S} T$-acyclic;

(2) $P \otimes_{S} T$ is $\operatorname{Hom}_{R}(T,-)$-acyclic.

In particular for cochain complexes $I^{\bullet}$ and $P^{\bullet}$ whose terms are injective right $R$ modules and projective right $S$-modules respectively, we have

$\mathbb{R} \operatorname{Hom}\left(T, I^{\bullet}\right) \otimes_{S}^{\mathbb{L}} T=\operatorname{Hom}\left(T, I^{\bullet}\right) \otimes_{S} T$ and $\mathbb{R} \operatorname{Hom}\left(T, P^{\bullet} \otimes_{S}^{\mathbb{L}} T\right)=\operatorname{Hom}\left(T, P^{\bullet} \otimes_{S} T\right)$. 
Finally, we recall that any adjoint pair of functors $(G, H)$ between categories of modules induces an adjoint pair $(\mathbb{L} G, \mathbb{R} H)$ between the associated unbounded derived categories. For other notation and results in derived categories we refer to 21, 29.

In the sequel we denote by $H$ the functor $\operatorname{Hom}_{R}(T,-)$ and by $G$ the functor $-\otimes_{S} T$.

Theorem 2.2. Let $T_{R}$ be a good n-tilting module and $S=\operatorname{End} T_{R}$. The following hold:

(1) The counit adjunction morphism

$$
\mathbb{L} G \circ \mathbb{R} H \rightarrow I d_{\mathcal{D}(R)}
$$

is invertible.

(2) The functor $\mathbb{R} H: \mathcal{D}(R) \rightarrow \mathcal{D}(S)$ is fully faithful.

(3) If $\Sigma$ is the system of morphisms $u \in \mathcal{D}(S)$ such that $\mathbb{L} G u$ is invertible in $\mathcal{D}(R)$, then $\Sigma$ admits a calculus of left fractions and the category $\mathcal{D}(S)\left[\Sigma^{-1}\right]$ coincides with the quotient category $\mathcal{D}(S)$ modulo the full triangulated subcategory $\operatorname{Ker}(\mathbb{L} G)$ of the objects annihilated by the functor $\mathbb{L} G$.

(4) There is a triangle equivalence

$$
\mathcal{D}(S)\left[\Sigma^{-1}\right] \underset{\mathbb{R} H}{\stackrel{\Theta}{\rightleftarrows}} \mathcal{D}(R),
$$

where $\Theta$ is the functor such that $\mathbb{L} G=\Theta \circ q$ with $q$ the canonical quotient functor $q: \mathcal{D}(S) \rightarrow \mathcal{D}(S)\left[\Sigma^{-1}\right]$.

Proof. (1) Let $M^{\bullet}$ be a complex in $\mathcal{D}(R)$ and consider a $K$-injective resolution $\underline{\mathbf{i}} M^{\bullet}$ of $M^{\bullet}$. By Corollary 2.1 we have

$$
\mathbb{L} G\left(\mathbb{R} H\left(M^{\bullet}\right)\right)=\mathbb{L} G\left(H\left(\underline{\mathbf{i}} M^{\bullet}\right)\right)=G\left(H\left(\underline{\mathbf{i}} M^{\bullet}\right)\right) .
$$

Since $\left(\operatorname{Hom}_{R}\left(T, I^{n}\right) \otimes_{S} T\right)_{n \in \mathbb{Z}}$ and $\underline{\mathbf{i}} M \bullet$ are isomorphic by Lemma 1.5. (2), we have

$$
\mathbb{L} G\left(\mathbb{R} H\left(M^{\bullet}\right)\right)=G\left(H\left(\underline{\mathbf{i}} M^{\bullet}\right)\right) \cong \underline{\mathbf{i}} M^{\bullet}=M^{\bullet} .
$$

Conditions (2), (3) and (4) follow by applying [15, Proposition I.1.3].

Let $\mathcal{C}$ be a triangulated category closed under arbitrary coproducts; recall that a triangle functor $L: \mathcal{C} \rightarrow \mathcal{C}$ is a Bousfield localization if there exists a natural transformation $\phi: 1_{\mathcal{C}} \rightarrow L$ such that for each $X$ in $\mathcal{C}$,

(1) $L\left(\phi_{X}\right): L(X) \rightarrow L^{2}(X)$ is an isomorphism;

(2) $L\left(\phi_{X}\right)=\phi_{L(X)}$.

In such a case the kernel $\mathcal{L}$ of $L$ is a full triangulated subcategory of $\mathcal{C}$ closed under coproducts; i.e. it is a localizing subcategory. The category

$$
\mathcal{L}_{\perp}:=\left\{X \in \mathcal{C}: \operatorname{Hom}_{\mathcal{C}}(\mathcal{L}, X)=0\right\}
$$

is called the subcategory of $\mathcal{L}$-local objects. If $\mathcal{L}_{\perp}$ is also closed under coproducts, then $\mathcal{L}$ is called smashing [8, 7].

A localization functor $L$ factorizes as

$$
\mathcal{C} \stackrel{q}{\rightarrow} \mathcal{C} / \operatorname{Ker} L \stackrel{\rho}{\cong} \mathcal{L}_{\perp} \stackrel{j}{\hookrightarrow} \mathcal{C},
$$


where $q$ is the canonical quotient functor and $\rho$ is an equivalence; $(\rho \circ q, j)$ is an adjoint pair. Moreover the composition

$$
\mathcal{L}_{\perp} \stackrel{j}{\rightarrow} \mathcal{C} \stackrel{q}{\rightarrow} \mathcal{C} / \operatorname{Ker} L
$$

is an equivalence and $(q, j \circ \rho)$ is an adjoint pair (see [7, Section 4], or [1, Proposition 1.6], or [23, Propositions 4.9.1, 4.11.1]).

We collect in the following theorem results appearing in [15] and [23, Section 4.9]. For the sake of completeness we include the proof.

Theorem 2.3. Let $(\Phi, \Psi)$ be an adjoint pair of covariant functors between triangulated categories

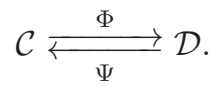

Denote by $\phi: 1_{\mathcal{C}} \rightarrow \Psi \circ \Phi$ and $\psi: \Phi \circ \Psi \rightarrow 1_{\mathcal{D}}$ the corresponding unit and counit. If $\psi$ is a natural isomorphism, then the functor $L:=\Psi \circ \Phi$ is a localization functor with kernel $\mathcal{L}=\operatorname{Ker} \Phi$. The functor $\Psi$ factorizes through $\mathcal{L}_{\perp}$ as $\Psi=j \circ \bar{\Psi}$, where $j$ is the inclusion $\mathcal{L}_{\perp} \hookrightarrow \mathcal{C}$. Finally we have a triangle equivalence

$$
\mathcal{L}_{\perp} \underset{\bar{\Phi}}{\stackrel{\Phi \circ j}{\rightleftarrows}} \mathcal{D}
$$

where $\Phi \circ j$ is the restriction of $\Phi$ to $\mathcal{L}_{\perp}$ and $\bar{\Psi}$ is the corestriction of $\Psi$ to $\mathcal{L}_{\perp}$.

Proof. Since $(\Phi, \Psi)$ is an adjoint pair, we have

$$
\psi_{\Phi(X)} \circ \Phi\left(\phi_{X}\right)=1_{\Phi(X)} ;
$$

applying the functor $\Psi$ we get

$$
\Psi\left(\psi_{\Phi(X)}\right) \circ L\left(\phi_{X}\right)=1_{L(X)} .
$$

On the other hand, again by the adjunction, we have

$$
\Psi\left(\psi_{\Phi(X)}\right) \circ \phi_{\Psi \Phi(X)}=1_{\Psi \Phi(X)} \text {, i.e. } \Psi\left(\psi_{\Phi(X)}\right) \circ \phi_{L(X)}=1_{L(X)} .
$$

Since $\psi_{\Phi(X)}$ is an isomorphism by assumption, we have that for each $X$ in $\mathcal{C}$,

$$
L\left(\phi_{X}\right)=\phi_{L(X)}=\left(\Psi\left(\psi_{\Phi(X)}\right)\right)^{-1}
$$

is an isomorphism. Hence $L$ is a localization functor.

An object $X$ belongs to $\mathcal{L}=\operatorname{Ker} L$ if and only if we have $0=\Phi(0)=\Phi(\Psi \Phi(X)) \cong$ $\Phi(X)$.

Next, since $L=\Psi \circ \Phi$ factorizes through $\mathcal{L}_{\perp}$ and $\Phi(\Psi(Y)) \cong Y$ for each $Y$ in $\mathcal{D}, \Psi$ also factorizes through $\mathcal{L}_{\perp}$. Therefore we have the following commutative diagram:

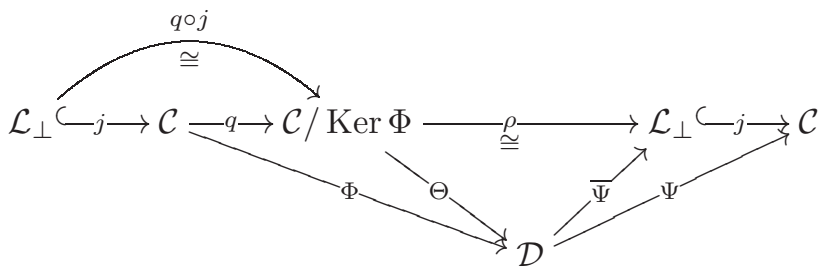

Finally $\Phi \circ j \circ \bar{\Psi}=\Phi \circ \Psi \cong 1_{\mathcal{D}}$, and $\bar{\Psi} \circ \Phi \circ j=\rho \circ q \circ j$, being a composition of two equivalences, is naturally isomorphic to $1_{\mathcal{L}_{\perp}}$.

Applying Theorem 2.3 to our context we obtain the following result. 
Corollary 2.4. Let $T_{R}$ be a good n-tilting $R$-module and $S=\operatorname{End}(T)$. Denoting by $\mathcal{E}$ the kernel of $\mathbb{L} G$, and denoting by $\mathbb{R} H$ and $\mathbb{L} G$ also their restriction and corestriction, we have a triangulated equivalence

$$
\mathcal{D}(R) \underset{\mathbb{L} G}{\stackrel{\mathbb{R} H}{\rightleftarrows}} \mathcal{E}_{\perp} .
$$

Embedding right $R$-modules and $S$-modules in $\mathcal{D}(R)$ and $\mathcal{D}(S)$ via the canonical functor, we obtain the following generalization of Miyashita's results [27, Theorem 1.16]:

Corollary 2.5. Let $T_{R}$ be a good n-tilting $R$-module and $S=\operatorname{End}(T)$. Then for each $0 \leq i \leq n$ there is an equivalence

$$
K E_{i} \underset{\operatorname{Tor}_{i}^{S}(-, T)}{\stackrel{\operatorname{Ext}_{R}^{i}(T,-)}{\leftrightarrows}} K T_{i} \cap \mathcal{E}_{\perp}
$$

Proof. Let $M \in K E_{i}$. Then by Corollary 2.4. $\mathbb{R} H(M)=R^{i} H(M)[-i]=$ $\operatorname{Ext}_{R}^{i}(T, M)[-i]$ belongs to $\mathcal{E}_{\perp}$. Since $\mathcal{E}_{\perp}$ is closed under shift, $\operatorname{Ext}_{R}^{i}(T, M) \in \mathcal{E}_{\perp}$. In $\mathcal{D}(R)$, by Theorem 2.2. (1), we have

$$
M \cong \mathbb{L} G \mathbb{R} H(M)=\mathbb{L} G\left(\operatorname{Ext}_{R}^{i}(T, M)[-i]\right) .
$$

Then for each $j \neq 0$,

$0=H^{j} \mathbb{L} G\left(\operatorname{Ext}_{R}^{i}(T, M)[-i]\right)=H^{j-i} \mathbb{L} G\left(\operatorname{Ext}_{R}^{i}(T, M)\right)=\operatorname{Tor}_{i-j}^{S}\left(\operatorname{Ext}_{R}^{i}(T, M), T\right)$.

Therefore $\operatorname{Ext}_{R}^{i}(T, M)$ belongs to $K T_{i} \cap \mathcal{E}_{\perp}$ and $M \cong \operatorname{Tor}_{i}^{S}\left(\operatorname{Ext}_{R}^{i}(T, M), T\right)$. Analogously if $N \in K T_{i} \cap \mathcal{E}_{\perp}$, then

$$
\mathbb{L} G(N)=L^{-i} G(N)[i]=\operatorname{Tor}_{i}^{S}(N, T)[i] ;
$$

and since $\mathbb{R} H \mathbb{L} G(N)=N$ in $\mathcal{D}(S)$, necessarily $\operatorname{Tor}_{i}^{S}(N, T)$ belongs to $K E_{i}$ and $N \cong \operatorname{Ext}_{R}^{i}\left(T, \operatorname{Tor}_{i}^{S}(N, T)\right)$.

Proposition 2.6. In the notation of Corollary 2.4, the following are equivalent:

(1) $T_{R}$ is a classical $n$-tilting;

(2) $\mathcal{E}=0$ or equivalently $\mathcal{E}_{\perp}=\mathcal{D}(S)$;

(3) the class $\mathcal{E}$ is smashing.

Proof. $(1 \Rightarrow 2)$. Let $N^{\bullet}$ be a complex in $\mathcal{E}$ and $\mathbf{p} N^{\bullet}$ a $K$-projective resolution of $N^{\bullet}$. By Lemma 1.5, (3) and (4), we have

$$
\begin{gathered}
0=\mathbb{R} H\left(\mathbb{L} G N^{\bullet}\right)=\mathbb{R} H\left(\mathbb{L} G \underline{\mathbf{p}} N^{\bullet}\right)=\mathbb{R} H\left(\underline{\mathbf{p}} N^{\bullet} \otimes_{S} T\right) \\
=\operatorname{Hom}_{R}\left(T, \underline{\mathbf{p}} N^{\bullet} \otimes_{S} T\right) \cong \underline{\mathbf{p}} N^{\bullet}=N^{\bullet} .
\end{gathered}
$$

We conclude that $\mathcal{E}=0$ by Corollary 2.4 .

$(2 \Rightarrow 3)$ is obvious.

$(3 \Rightarrow 2)$. Since $S=\mathbb{R} H\left(T_{R}\right), \mathcal{E}_{\perp}$ contains the bounded complexes of finitely generated projective $S$-modules; that is, $\mathcal{E}_{\perp}$ contains the set $\mathcal{T}^{c}$ of the compact objects of $\mathcal{D}(S)$.

Since $\mathcal{D}(S)$ is compactly generated by $\mathcal{T}^{c}, \mathcal{D}(S)$ is the smallest triangulated category closed under coproducts and containing $\mathcal{T}^{c}$. Thus, if $\mathcal{E}_{\perp}$ is closed under coproducts, we get that $\mathcal{E}_{\perp}=\mathcal{D}(S)$; hence $\mathcal{E}=0$.

$(2 \Rightarrow 1)$. By Corollary 2.4 , condition (2) implies that $\mathbb{L} G$ induces an equivalence between $\mathcal{D}(S)$ and $\mathcal{D}(R)$. Hence by [18, or [22, Section 4.1], $T_{R}$ is a classical $n$-tilting module. 


\section{REFERENCES}

[1] L. Alonso Tarrío, A. Jeremías López, and M. J. Souto Salorio. Localization in categories of complexes and unbounded resolutions. Canad. J. Math., 52(2):225-247, 2000. MR 1755776 (2001i:18019)

[2] L. Angeleri Hügel and J. Sánchez. Tilting modules arising from ring epimorphisms. Algebr. Represent. Theory, 14:217-246, 2011.

[3] L. Angeleri-Hügel and J. Trlifaj. Tilting theory and the finitistic dimension conjectures. Trans. Amer. Math. Soc., 354(11):4345-4358 (electronic), 2002. MR1926879 (2003j:16012)

[4] S. Bazzoni. A characterization of $n$-cotilting and $n$-tilting modules. J. Algebra, 273(1):359372, 2004. MR2032465 (2005h:16017)

[5] S. Bazzoni. Equivalences induced by infinitely generated tilting modules. Proc. Amer. Math. Soc., 138(2):533-544, 2010. MR2557170 (2011a:16012)

[6] I. N. Bernšteln, I. M. Gel'fand, and V. A. Ponomarev. Coxeter functors, and Gabriel's theorem. Uspehi Mat. Nauk, 28(2(170)):19-33, 1973. MR0393065 (52:13876)

[7] M. Bökstedt and A. Neeman. Homotopy limits in triangulated categories. Compositio Math., 86(2):209-234, 1993. MR.1214458 (94f:18008)

[8] A. K. Bousfield. The localization of spectra with respect to homology. Topology, 18(4):257281, 1979. MR551009 (80m:55006)

[9] S. Brenner and M. C. R. Butler. Generalizations of the Bernstein-Gel'fand-Ponomarev reflection functors. In Representation theory, II (Proc. Second Internat. Conf., Carleton Univ., Ottawa, Ont., 1979), volume 832 of Lecture Notes in Math., pages 103-169. Springer, Berlin, 1980. MR607151 (83e:16031)

[10] E. Cline, B. Parshall, and L. Scott. Derived categories and Morita theory. J. Algebra, 104(2):397-409, 1986. MR866784 (88a:16075)

[11] R. Colpi and J. Trlifaj. Tilting modules and tilting torsion theories. J. Algebra, 178(2):614634, 1995. MR.1359905 (97e:16003)

[12] A. Facchini. A tilting module over commutative integral domains. Comm. Algebra, 15(11):2235-2250, 1987. MR912770 (89c:13028)

[13] A. Facchini. Divisible modules over integral domains. Ark. Mat., 26(1):67-85, 1988. MR 948281 (90a:13018)

[14] L. Fuchs. On divisible modules over domains. In Abelian groups and modules (Udine, 1984), volume 287 of CISM Courses and Lectures, pages 341-356. Springer, Vienna, 1984. MR789830 (86j:13012)

[15] P. Gabriel and M. Zisman. Calculus of fractions and homotopy theory. Ergebnisse der Mathematik und ihrer Grenzgebiete, Band 35. Springer-Verlag New York, Inc., New York, 1967. MR0210125 (35:1019)

[16] R. Göbel and J. Trlifaj. Approximations and endomorphism algebras of modules, volume 41 of de Gruyter Expositions in Mathematics. Walter de Gruyter GmbH \& Co. KG, Berlin, 2006. MR2251271 (2007m:16007)

[17] E. Gregorio and A. Tonolo. Weakly tilting bimodules. Forum Math., 13(5):589-614, 2001. MR $1858490(2002 \mathrm{i}: 16008)$

[18] D. Happel. On the derived category of a finite-dimensional algebra. Comment. Math. Helv., 62(3):339-389, 1987. MR910167(89c:16029)

[19] D. Happel and C. M. Ringel. Tilted algebras. Trans. Amer. Math. Soc., 274(2):399-443, 1982. MR675063(84d:16027)

[20] D. K. Harrison. Infinite abelian groups and homological methods. Ann. of Math. (2), 69:366391, 1959. MR0104728(21:3481)

[21] R. Hartshorne. Residues and duality. Lecture notes of a seminar on the work of A. Grothendieck, given at Harvard, 1963/64. With an appendix by P. Deligne. Lecture Notes in Mathematics, No. 20. Springer-Verlag, Berlin, 1966. MR0222093 (36:5145)

[22] B. Keller. On the construction of triangle equivalences. Lectures Workshop Pappenheim 1994, 1994.

[23] H. Krause. Localization theory for triangulated categories. Proceedings of "Workshop on Triangulated Categories" (Leeds, 2006), vol. 375 of London Math. Soc. Lecture Note Ser., pages 161-235. Cambridge Univ. Press, Cambridge, 2010.

[24] F. Lukas. Infinite-dimensional modules over wild hereditary algebras. J. London Math. Soc. (2), 44(3):401-419, 1991. MR:1149004 (93b:16021) 
[25] F. Mantese and A. Tonolo. Reflexivity in derived categories. Forum Math., 22(6):1161-1191, 2010.

[26] E. Matlis. Cotorsion modules. Mem. Amer. Math. Soc. No., 49:66, 1964 . MR0178025 $(31: 2283)$

[27] Y. Miyashita. Tilting modules of finite projective dimension. Math. Z., 193(1):113-146, 1986. MR852914 (87m:16055)

[28] I. Reiten and C. M. Ringel. Infinite dimensional representations of canonical algebras. Canad. J. Math., 58(1):180-224, 2006. MR2195596(2006k:16006)

[29] C. A. Weibel. An introduction to homological algebra, volume 38 of Cambridge Studies in Advanced Mathematics. Cambridge University Press, Cambridge, 1994. MR 1269324|(95f:18001)

Dipartimento di Matematica Pura ed Applicata, Università di Padova, via Trieste 63, I-35121 PAdova, ItAly

E-mail address: bazzoni@math.unipd.it

Dipartimento di Informatica, Università degli Studi di Verona, strada Le Grazie 15, I-37134 VERONA, ItALY

E-mail address: francesca.mantese@univr.it

Dipartimento di Matematica Pura ed Applicata, Università di Padova, via Trieste 63, I-35121 PADOVA, ItALy

E-mail address: tonolo@math.unipd.it 